\title{
Preoperative Temporary Discontinuation of Aspirin Medication Does Not Increase the Allogeneic Transfusion Rate and Blood Loss in Primary Total Hip Arthroplasty
}

\author{
Myung-Rae Cho, MD, Chung Mu Jun, MD, Won-Kee Choi, MD \\ Department of Orthopaedic Surgery, Daegu Catholic University College of Medicine, Daegu, Korea
}

Purpose: This study aims to determine whether preoperative temporary discontinuation of aspirin (100 mg/d) use is a safe procedure does not increase blood loss and the need for transfusion after total hip arthroplasty (THA). Materials and Methods: This study retrospectively reviewed 219 patients who underwent consecutive primary THA from January 2012 to December 2018. They were divided into the experimental group (42 cases) that discontinued aspirin intake 7 days before surgery and the control group (150 cases) that had no history of use of antiplatelet agents. To compare initial blood loss between the two groups, we analyzed the changes hemoglobin $(\mathrm{Hb})$ levels and hematocrit values measured preoperatively and in lowest values measured during three days after surgery. In addition, transfusion rate was compared within the first two postoperative weeks between the two groups. A multiple logistic regression was performed to assess the association of transfusion with age, gender, use of antiplatelet agents, preoperative anesthetic risk, body weight and preoperative $\mathrm{Hb}$.

Results: No statistically significant difference was found in the changes in lowest $\mathrm{Hb}$ level $(P=0.30)$ and hematocrit value $(P=0.14)$ measured preoperatively and for three days after surgery between the experimental group and the control group. There was no statistically significant association between transfusion and the use of antiplatelet agents, and preoperative $\mathrm{Hb}$ level was identified as a factor that affected the need for transfusion (odds ratio, $0.427 ; P=0.001$ ).

Conclusion: Preoperative temporary discontinuation of aspirin use for 7 days before surgery did not increase initial blood loss after THA and the need for transfusion in the first two postoperative weeks compared to patients with no history of use of antiplatelet agents.

Key Words: Hip replacement arthroplasty, Blood transfusion, Aspirin

Submitted: February 4, 2019 1st revision: April 26, 2019

2nd revision: May 8, 2019 Final acceptance: May 9, 2019

Address reprint request to

Won-Kee Choi, MD

(https://orcid.org/0000-0002-4671-5656)

Department of Orthopaedic Surgery, Daegu Catholic University

Medical Center, 33 Duryugongwon-ro 17-gil, Nam-gu, Daegu

42472, Korea

TEL: +82-53-650-4277 FAX: +82-53-652-4276

E-mail: cwk1009dahanmail.net
This is an Open Access article distributed under the terms of the Creative Commons Attribution Non-Commercial License (http://creativecommons. org/licenses/by-nc/4.0) which permits unrestricted non-commercial use, distribution, and reproduction in any medium, provided the original work is properly cited. 
Myung-Rae Cho et al. Temporary Discontinuation of Aspirin Does Not Increase Blood Loss in THA

\section{INTRODUCTION}

Rates of aspirin use are increasing for primary and secondary prevention of cardiovascular diseases ${ }^{1}$. With this recent trend, rates of total hip arthroplasty (THA) are also increasing in patients on aspirin therapy. When performing THA, surgeons must decide whether or not to discontinue antiplatelet therapy after carefully considering risks associated with aspirin use and the likelihood of aggravation of comorbid diseases ${ }^{2}$. Since 2011, the clinical practice guidelines of the American Academy of Orthopedic Surgeons (AAOS) have recommended temporary withdrawal of antiplatelet drugs including aspirin when performing lower limb arthroplasty as an elective operation ${ }^{3}$. Although recent studies have addressed that the continuation of aspirin will not increase the risk of blood loss and the need for allogeneic transfusion after $\mathrm{THA}^{4}$, , surgeons have not yet reached a consensus on this issue. In our institution, THA is performed after discontinuation of antiplatelet agents including aspirin for 7 days before surgery through interdisciplinary care with the Division of Internal Medicine, unless patients were included in the high-risk group. However, preoperative temporary discontinuation of aspirin use still remains controversial because very few clinical studies have been conducted on whether temporary cessation of aspirin would decrease blood loss and transfusion rate after THA. We compared the first postoperative transfusion and allogeneic transfusion during the first two postoperative weeks between patients who temporarily stopped taking aspirin before surgery and those who had no history of aspirin use by retrospectively reviewing cases that had undergone consecutive primary THA by the same surgeon after visiting our hospital outpatiently from January 2012 to December 2018. We aimed to determine whether preoperative temporary discontinuation of aspirin use is a safe procedure that does not increase initial blood loss and the need for allogeneic transfusion after THA. We hypothesized that there would be no difference in allogeneic transfusion rates in the first two postoperative weeks, but there would be a difference in the indices ${ }^{5)}$ of blood loss between the two groups with withdrawal of aspirin therapy 7 days before elective surgery and no history of aspirin.

\section{MATERIALS AND METHODS}

This study retrospectively reviewed the electronic medical record data of 219 patients who underwent consecutive primary THA by a single surgeon at a single institution from January 2012 to December 2018. Of these 219 patients who had undergone THA as elective surgery, except for those who had undergone the procedure following hip fracture, we excluded 19 patients who used antiplatelet agents other than aspirin and anticoagulants, 5 who had a hemorrhagic history, and 3 who decided to continue aspirin therapy after being referred to the Division of Internal Medicine. A total of 192 patients were finally chosen as the subjects and they were divided into the experimental group (42 cases) that discontinued aspirin intake 7 days before surgery and the control group (150 cases) that had no history of use of antiplatelet agents including aspirin. To compare allogeneic transfusion rate and initial blood loss during the patient's hospital stay between the two groups, we analyzed the changes in lowest hemoglobin $(\mathrm{Hb})$ levels and hematocrit values measured preoperatively and for three days after surgery. In addition, allogeneic transfusion rate was compared within the first two postoperative weeks between the two groups. The study was approved by the Institutional Review Board of Daegu Catholic University College of Medicine (CR-19-015).

All operations were carried out by a single surgeon using the modified Hardinge approach. A drainage catheter was inserted after surgery in all patients. The catheter was removed on the first or second postoperative day depending on the amount of drainage. On the first postoperative day, patients who stopped taking aspirin treatment before surgery resumed their regular aspirin regimen. When performing THA, medication was not given for prevention of deep vein thrombosis (DVT), unless patients had a history of DVT or was in the high-risk group for DVT.

\section{Allogeneic Transfusion Rates}

The frequency of allogeneic transfusion was measured during two weeks of hospital stay after THA. Based on the clinical guidelines of the American Association of Blood Banks, the indications for blood transfusion were an $\mathrm{Hb}$ level of $8 \mathrm{~g} / \mathrm{dL}$ or less and patient's clinical symptoms ${ }^{6}$.

\section{Difference in Blood Loss}

Changes in hematocrit and $\mathrm{Hb}$ levels, the two important indicators in measuring blood loss, were assessed and compared $^{5}$. Changes in the minimal $\mathrm{Hb}$ levels and hematocrit values measured preoperatively and for three days after surgery were analyzed to assess the initial blood loss after surgery. 


\section{Hip \& Pelvis}

Hip Pelvis 31(2): 82-86, 2019

Table 1. Patient's Preoperational Epidemiology

\begin{tabular}{lccc}
\hline \hline Variable & Holder of aspirin $(\mathrm{n}=42)$ & No history of aspirin $(\mathrm{n}=150)$ & $P$-value \\
\hline Sex, male:female & $21: 21$ & $101: 49$ & 0.03 \\
Age $(\mathrm{yr})$ & $67.71 \pm 7.52$ & $59.89 \pm 12.21$ & 0.01 \\
Height $(\mathrm{cm})$ & $159.84 \pm 7.18$ & $163.85 \pm 7.79$ & 0.01 \\
Weight $(\mathrm{kg})$ & $60.57 \pm 9.92$ & $63.53 \pm 9.25$ & 0.07 \\
ASA classification & $36: 6(\mathrm{G} 2: \mathrm{G} 3)$ & $34: 110: 6(\mathrm{G} 1: \mathrm{G} 2: \mathrm{G} 3)$ & 0.01 \\
Hemoglobin $(\mathrm{g} / \mathrm{dL})$ & $12.18 \pm 1.69$ & $12.69 \pm 1.51$ & 0.06 \\
Hematocrit $(\%)$ & $36.21 \pm 4.76$ & $38.20 \pm 4.27$ & 0.01 \\
\hline
\end{tabular}

Values are presented as number only or mean \pm standard deviation.

ASA: American Society of Anesthesiologists, G: grade.

\section{Statistical Analysis}

A $t$-test was used for comparing changes in $\mathrm{Hb}$ and hematocrit levels that reflected the need for allogeneic transfusion and blood loss between the two groups. A chi-square test was used to explore the association between antiplatelet therapy until 7 days before surgery and allogeneic transfusion after surgery. A multiple logistic regression was performed to assess the association of the need for allogeneic transfusion with patient's age, gender, use of antiplatelet drugs, preoperative anesthetic risk (American Society of Anesthesiologists [ASA] classification), and weight and preoperative $\mathrm{Hb}$ level. $P$-values of less than 0.05 were considered statistically significant. Statistical analysis was performed using the IBM SPSS Statistics ver. 19 (IBM Corp., Armonk, NY, USA).

\section{RESULTS}

\section{Epidemiology of Patients}

The most common diagnosis of patients was avascular necrosis of the femoral head, followed by degenerative and post-traumatic arthritis, rheumatoid arthritis, ankylosing spondylitis and others. All patients received the same non-cemented cup as an acetabular component. Femoral components used were all non-cemented stem except in 2 cases of cemented in the aspirin group and a 1 case of cemented in the non-aspirin group. The aspirin group had statistically significantly more number of females, was older in age and smaller in height, and had higher ASA scores and lower preoperative hematocrit $(P=0.01)$ compared to the non-aspirin group (Table 1).
Table 2. The Result of Transfusion Rates between Two Groups

\begin{tabular}{lccc}
\hline \hline \multirow{2}{*}{ Transfusion } & \multicolumn{2}{c}{ Aspirin } & P-value \\
\cline { 2 - 3 } & No history & Holder & \\
\hline No $(\mathrm{n}=121)$ & 97 & 24 & 0.37 \\
Yes $(\mathrm{n}=71)$ & 53 & 18 & \\
Total $(\mathrm{n}=192)$ & 150 & 42 & \\
\hline
\end{tabular}

\section{Allogeneic Transfusion}

Allogeneic transfusion was performed in 71 (37.0\%) of 192 patients within the first two postoperative weeks. Of these patients, there were 18 of 42 patients with aspirin use and 53 of 150 with no history of aspirin medication. No statistically significant difference was shown between the two groups $(P=0.37)$. The decision to give allogeneic transfusion was made intraoperatively in $10(5.2 \%)$ of 192 patients. These 10 cases included 7 of 42 cases with temporary discontinuation of aspirin medication and 3 of 150 cases with no history of aspirin use. The likelihood of and need for allogeneic transfusion during surgery was higher in the group with a history of aspirin use $(P=0.001)$.

A chi-square test revealed that no statistically significant difference was found between aspirin intake until 7 days before surgery and allogeneic transfusion after surgery (Table 2). A multiple logistic regression was performed to assess the association of allogeneic transfusion with multifactorial variables including patient's age, gender, use of antiplatelet agents, preoperative anesthetic risk (ASA), weight, and preoperative $\mathrm{Hb}$ level. As results, there was no statistically significant association between allogeneic transfusion and the use of antiplatelet agents, and preoperative $\mathrm{Hb}$ level was identified as a factor that affected the need for allogeneic transfusion (Table 3). 
Hip \& Pelvis

Myung-Rae Cho et al. Temporary Discontinuation of Aspirin Does Not Increase Blood Loss in THA

Table 3. Analysis of Multiple Variables Effects for Transfusion Rates

\begin{tabular}{|c|c|c|c|c|c|c|}
\hline \multirow{2}{*}{ Variable } & \multicolumn{3}{|c|}{ Crude } & \multicolumn{3}{|c|}{ Adjusted } \\
\hline & OR & $95 \% \mathrm{Cl}$ & $P$-value & OR & $95 \% \mathrm{Cl}$ & $P$-value \\
\hline History of aspirin & 0.729 & $0.363-1.462$ & 0.373 & 1.445 & $0.604-3.457$ & 0.408 \\
\hline Sex & & & & 1.136 & $0.520-2.482$ & 0.749 \\
\hline Age & & & & 1.017 & $0.981-1.054$ & 0.355 \\
\hline Weight & & & & 0.986 & $0.945-1.029$ & 0.515 \\
\hline ASA & & & & 2.981 & $0.774-11.486$ & 0.112 \\
\hline Preop hemoglobin & 0.396 & $0.297-0.528$ & 0.001 & 0.427 & $0.316-0.575$ & 0.001 \\
\hline
\end{tabular}

OR: odds ratio, $\mathrm{Cl}$ : confidence interval, ASA: American Society of Anesthesiologists, Preop: preoperative.

OR of history of aspirin: no history/aspirin holder; OR of sex: male/female; OR of ASA: (Grade 2+Grade 3)/Grade 1.

Table 4. Changes of Hemoglobin, Hematocrit at the Postoperation between the Two Groups

\begin{tabular}{lccc}
\hline \hline Difference & Holder of aspirin $(\mathrm{n}=35)$ & No history of aspirin $(\mathrm{n}=147)$ & $P$-value \\
\hline Hemoglobin & $2.60 \pm 1.20$ & $2.81 \pm 0.99$ & 0.30 \\
Hematocrit & $7.69 \pm 3.38$ & $8.58 \pm 3.15$ & 0.14 \\
\hline
\end{tabular}

\section{Comparison of Blood Loss and Drainage}

Changes in lowest $\mathrm{Hb}$ and hematocrit levels measured preoperatively and for three days after surgery were compared because these values indirectly represented blood loss. As results, there was no statistically significant difference in the changes in lowest $\mathrm{Hb}$ and hematocrit values between the two groups with aspirin use until 7 days before surgery and no history of aspirin use (Table 4). Drainage volume was $305 \mathrm{~mL}$ in the aspirin group and $314 \mathrm{~mL}$ in the non-aspirin group, showing no statistically significant difference $(P=0.82)$.

\section{DISCUSSION}

In the present study, although patients who preoperatively took aspirin were more likely to have lower hematocrit before surgery compared to those who did not use aspirin, we found out that the use of aspirin did not increase blood loss and the need for allogeneic transfusion after THA within the first two postoperative weeks compared to the group without any history of aspirin use.

Temporary discontinuation of aspirin for 7 days before THA had demonstrated no difference in the potential risk of bleeding compared with patients with no history of aspirin use.

In our study, $37.0 \%$ of the subjects received allogeneic blood transfusion during the first two weeks after surgery. Transfusion rates vary by surgeon, ranging from $4 \%$ to
$68 \% \%^{7}$. However, the problem is that allogeneic transfusion following THA can cause many side effects including postoperative infection ${ }^{8)}$. For this reason, surgeons try to minimize transfusion ${ }^{9}$. However, surgeons need to reach a consensus on aspirin use because THA rates and populations taking antiplatelet agents for primary and secondary prevention of cardiovascular diseases are gradually increasing. There still remain controversial issues, and many institutions still recommend temporary withdrawal of aspirin use before surgery. Surgeons usually ask patients to stop aspirin therapy 7 days prior to surgery, because the average lifespan of the platelet is 7 to 10 days ${ }^{10}$. However, there are almost no studies on the comparison of transfusion rates and blood loss between patients with temporary discontinuation of aspirin use and those without a history of aspirin.

In this study, transfusion rate was high in patients with older age, lower height and weight, low preoperative $\mathrm{Hb}$ levels, and high ASA scores. There was no statistically significant difference in gender and aspirin use 7 days before surgery between the two groups. These outcomes were comparable to those of Hart et al. ${ }^{7}$ study on the risk factors of allogeneic blood transfusion following THA.

In our study, the initial blood loss after surgery did not increase and a statistically significant difference was not found in allogeneic transfusion rates within the first two weeks after surgery in the group that temporarily stop taking aspirin compared to the group that had no history of aspirin intake. A number of studies obtained the same 
findings that the initial blood loss after THA did not increase in the low-dose aspirin group compared to the non-aspirin group ${ }^{11}$.

A large number of studies reported that low $\mathrm{Hb}$ level and hematocrit before surgery increased the risk of allogeneic transfusion after surgery ${ }^{9}$. In the current study, statistically significant low hematocrit was shown in the group with aspirin discontinuation and $\mathrm{Hb}$ level was somewhat lower. These findings revealed that the past history of aspirin use itself may increase the risk of allogeneic transfusion after surgery. In our study, allogeneic transfusion was performed intraoperatively in 10 of 192 patients. These 10 cases included 7 of 42 cases with temporary discontinuation of aspirin and 3 of 150 cases with no history of aspirin use. The likelihood of and need for intraoperative transfusion were greater in the group with previous aspirin use $(P=$ $0.001)$.

There are several limitations to note in the present study. First, this study is limited by the retrospective nature of the analysis. Second, this study involved patients with temporary withdrawal of aspirin use. Temporary discontinuation of antiplatelet agents has an advantage of reducing blood loss, but can also incur serious adverse effects due to the increased risk of thrombotic events caused by platelet rebound effect ${ }^{12}$. In our study, pulmonary thromboembolism occurred in one of patients with temporary suspension of aspirin and two of patients without a history of aspirin therapy. However, we did not perform a thorough investigation because we assumed that data collection was inaccurate due to the retrospective nature of the analysis.

\section{CONCLUSION}

Despite temporary discontinuation of aspirin, patients with a history of aspirin use were more likely to have lower preoperative $\mathrm{Hb}$ and hematocrit levels compared to those with no history of aspirin use, and they had a statistically significantly higher rate of intraoperative transfusion $(P=0.001)$. Preoperative temporary discontinuation of aspirin for 7 days before surgery did not increase initial blood loss after THA and the need for allogeneic transfusion in the first two postoperative weeks compared to patients with no history of use of antiplatelet agents.

\section{CONFLICT OF INTEREST}

The authors declare that there is no potential conflict of interest relevant to this article.

\section{REFERENCES}

1. Nansseu JR, Noubiap JJ. Aspirin for primary prevention of cardiovascular disease. Thromb J. 2015;13:38.

2. Douketis JD, Spyropoulos AC, Spencer FA, et al. Perioperative management of antithrombotic therapy: Antithrombotic Therapy and Prevention of Thrombosis, 9th ed: American College of Chest Physicians Evidence-Based Clinical Practice Guidelines. Chest. 2012;141(2 Suppl):e326S-50S.

3. Mont MA, Jacobs JJ. AAOS clinical practice guideline: preventing venous thromboembolic disease in patients undergoing elective hip and knee arthroplasty. J Am Acad Orthop Surg. 2011;19:777-8.

4. Meier R, Marthy R, Saely CH, Kuster MS, Giesinger K, Rickli H. Comparison of preoperative continuation and discontinuation of aspirin in patients undergoing total hip or knee arthroplasty. Eur J Orthop Surg Traumatol. 2016; 26:921-8.

5. Gross JB. Estimating allowable blood loss: corrected for dilution. Anesthesiology. 1983;58:277-80.

6.American Society of Anesthesiologists Task Force on Perioperative Blood Transfusion and Adjuvant Therapies. Practice guidelines for perioperative blood transfusion and adjuvant therapies: an updated report by the American Society of Anesthesiologists Task Force on Perioperative Blood Transfusion and Adjuvant Therapies. Anesthesiology. 2006; 105:198-208.

7. Hart A, Khalil JA, Carli A, Huk O, Zukor D, Antoniou J. Blood transfusion in primary total hip and knee arthroplasty. Incidence, risk factors, and thirty-day complication rates. $J$ Bone Joint Surg Am. 2014;96:1945-51.

8. Friedman R, Homering M, Holberg G, Berkowitz SD. Allogeneic blood transfusions and postoperative infections after total hip or knee arthroplasty. J Bone Joint Surg Am. 2014;96:272-8.

9. Lee JH, Han SB. Patient blood management in hip replacement arthroplasty. Hip Pelvis. 2015;27:201-8.

10. Harker LA. The kinetics of platelet production and destruction in man. Clin Haematol. 1977;6:671-93.

11. Cossetto DJ, Goudar A, Parkinson K. Safety of peri-operative low-dose aspirin as a part of multimodal venous thromboembolic prophylaxis for total knee and hip arthroplasty. J Orthop Surg (Hong Kong). 2012;20:341-3.

12. Ford I. Coming safely to a stop: a review of platelet activity after cessation of antiplatelet drugs. Ther Adv Drug Saf. 2015;6:141-50. 